\title{
BMJ Open Procalcitonin-guided initiation of antibiotics in AECOPD inpatients: study protocol for a multicenter randomised controlled trial
}

\author{
Lixue Huang (D) , ${ }^{1,2}$ Jinxiang Wang, ${ }^{3}$ Xiaoying Gu, ${ }^{2,4}$ Weili Sheng, ${ }^{5}$ Yeming Wang, ${ }^{1,2}$ \\ Bin $\mathrm{Cao}^{1,2,6,7}$
}

To cite: Huang L, Wang J, Gu X, et al. Procalcitoninguided initiation of antibiotics in AECOPD inpatients: study protocol for a multicenter randomised controlled trial. BMJ Open 2021;11:e049515. doi:10.1136/ bmjopen-2021-049515

- Prepublication history and additional supplemental material for this paper are available online. To view these files, please visit the journal online. (http://dx.doi.org/10.1136/ bmjopen-2021-049515).

Received 27 January 2021 Accepted 19 July 2021

Check for updates

(C) Author(s) (or their employer(s)) 2021. Re-use permitted under CC BY-NC. No commercial re-use. See rights and permissions. Published by BMJ.

For numbered affiliations see end of article.

Correspondence to

Dr Bin Cao;

caobin_ben@163.com

\section{ABSTRACT}

Introduction Current antibiotic prescription for acute exacerbation of chronic obstructive pulmonary disease (AECOPD) is generally based on the Anthonisen criteria in The Global Initiative for Chronic Obstructive Pulmonary Disease (GOLD) guideline that have a potential risk of antibiotics overuse. The dilemma is to identify patients who are most likely to benefit from antibiotics while avoiding unnecessary antibiotic use. Procalcitonin (PCT), a more sensitive and specific biomarker of bacterial infection than other conventional laboratory tests, has the potential to determine those patients in whom antibiotics would be beneficial. It is unclear whether PCT-guided antibiotic therapy is safe and effective for patients hospitalised with AECOPD. The study hypothesis is that PCT-guided antibiotic therapy could reduce the antibiotic prescription rate for AECOPD, compared with the GOLD guideline recommendations, without negatively impacting the treatment success rate.

Methods and analysis In this multicenter, open-label, randomised controlled trial, we aim to enrol 500 hospitalised patients with AECOPD that will be randomly assigned to either a PCT-guided group or a GOLD guideline-guided group. The coprimary endpoints are antibiotic prescription rate for $A E C O P D$ within 30 days post randomisation and treatment success rate at day 30 post randomisation. The secondary outcomes include: antibiotic prescription rate at day 1 post randomisation; hospital antibiotic exposure; length of hospital stay; rate of subsequent exacerbation and hospital readmission; overall mortality within 30 days post randomisation; changes in lung function and the score of COPD assessment test and modified Medical Research Council; and rate of intensive care unit admission.

Ethics and dissemination This trial has been approved by the ethic committee of China-Japan Friendship Hospital. The findings of the study will be disseminated in peer-reviewed journals. If the results of the study are positive, PCT-guided antibiotic therapy is likely to change the guidelines for antibiotic recommendations for patients with AECOPD.

Trial registration number ClinicalTrials.gov: NCT04682899.

\section{INTRODUCTION}

In China, COPD is the third leading cause of death ${ }^{1}$ and the overall prevalence in adults
Strengths and limitations of this study

This is a nationwide, multicenter, randomised controlled trial in China.

- The study design conclude two primary outcomes regarding safety and effectivity.

- The study encompasses multiple clinically related secondary outcomes.

- The study has a sample size large enough to provide high-quality evidence to evaluate the safety and effectivity of procalcitonin in patients with acute exacerbation of chronic obstructive pulmonary disease.

- One limitation of the study is that patients, primary clinicians and laboratory staff are clear to the grouping, only outcomes assessor and statisticians are blinded.

aged 40 years or older has risen from $8.2 \%$ (during 2002-2004) ${ }^{2}$ to $13.7 \%$ (during 20122015). Acute exacerbations are the most common reasons for hospitalisation and death for patients with COPD. The Global Initiative for Chronic Obstructive Pulmonary Disease (GOLD) guideline notes that exacerbations of COPD are mainly triggered by respiratory viral infections ${ }^{4}$; however, a very high proportion of patients with acute exacerbation of COPD (AECOPD) received antibiotic treatment. The prescription rate of antibiotics for inpatients with AECOPD in the USA, Europe and China all exceeds $85 \% .^{5-7}$ This brings a high risk of unnecessary use of antibiotics. Antibiotics overuse wastes medical resources, drives antimicrobial resistance, may cause side effects, negatively affects the microbiome of patients and distracts from potentially more effective interventions. ${ }^{8}$

The current GOLD guidelines recommended antibiotics prescription in patients of Anthonisen I and II $^{9}$ with sputum purulence, as well as patients with mechanical ventilation. ${ }^{10}$ The GOLD guidelines assume 
purulence of sputum indicates bacterial infection; however, previous trials have shown that it was not a reliable marker for bacterial presence. ${ }^{11} 12$ On the basis of guideline recommendations, antibiotic prescription should be implemented on all patients with AECOPD receiving mechanical ventilation. This can obviously lead to overuse of antibiotics, particularly in those with acute exacerbation driven by viral infection or environmental factors. Thus, wide implementation of the antibiotic recommendations from the GOLD guidelines has the potential risk of antibiotic overuse.

Identifying AECOPD driven by bacterial infection is necessary prior to antibiotics prescription. Procalcitonin (PCT), a reliable biomarker of bacterial infection, has the potential to guide the prescription of antibiotics. Several randomised controlled trials (RCTs) have explored the effect of PCT on guiding antibiotic therapy in patients with lower respiratory infections ${ }^{13-15}$ and especially patients with AECOPD. ${ }^{16}$ Given the limitations of these studies, whether PCT-guided antibiotic therapy will safely and effectively bring clinical benefit for AECOPD was unclear. PCT guidance, markedly and safely, reduced antibiotic prescriptions or the duration of antibiotic therapy in patients with lower respiratory infections. ${ }^{13-15}$ However, enrolled patients were not only restricted to patients with COPD, but also included patients with community acquired pneumonia, asthma and bronchitis. An RCT study of 208 inpatients with AECOPD showed that PCT guidance resulted in reduced antibiotic prescription and exposure compared with standard care therapy, with no difference in secondary outcomes (including success rate, subsequent exacerbation and rehospitalisation rates) between the two groups. ${ }^{16}$ However, the sample size was calculated according to a single outcome of antibiotic prescription rate and this limited sample size (208 AECOPD inpatients) was insufficiently powered to show whether PCT-guided algorithms do not affect secondary outcomes. Due to the lack of confirmatory PCT trials with rigorous methodology for COPD, the current GOLD guideline still recommend antibiotic prescription according to the Anthonisen criteria of $1987 .{ }^{9}$

Evidence from our previous study has shown that patients with AECOPD and with a PCT level less than $0.1 \mathrm{ng} / \mathrm{mL}$ did not benefit from the additional antibiotic therapy. ${ }^{17}$ In this protocol, we aim to conduct a multicenter RCT to determine the effect of PCT-guided antibiotic therapy compared with the current GOLD guideline recommendations in patients with AECOPD.

\section{METHODS/DESIGN Objective}

The primary aim is to determine whether PCT-guided antibiotic therapy will reduce the antibiotic prescription rate for AECOPD without negatively impacting the treatment success rate, compared with the GOLD guideline recommendations.
Hospitalized patients with AECOPD

Screening

Eligible participants

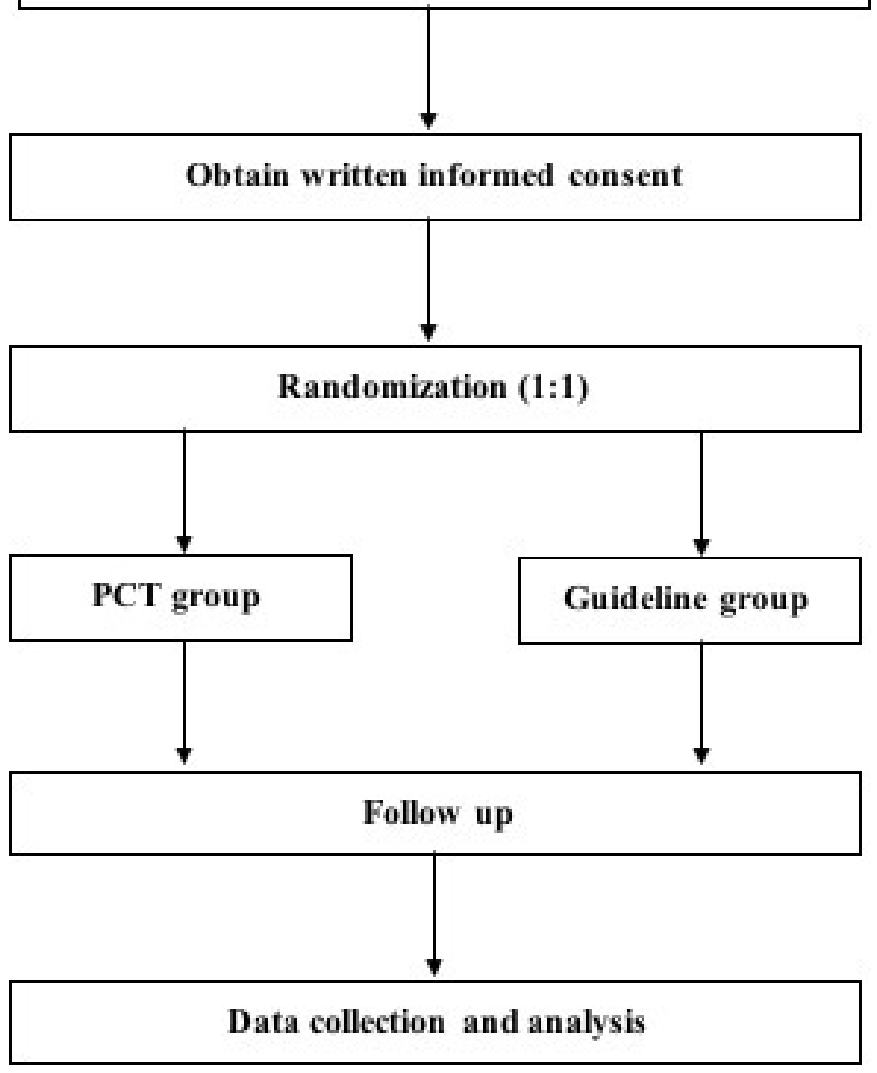

Figure 1 The flow chart of enrolled participants. AECOPD, acute exacerbation of chronic obstructive pulmonary disease; PCT, procalcitonin.

\section{Design}

This is a nationwide, multicenter, open-label, RCT that will be conducted at 10 hospitals in China. Eligible participants will be randomly assigned to either the PCT-guided antibiotic therapy (PCT group) or the GOLD guideline antibiotic recommendations (guideline group) in a ratio of 1:1. Figure 1 shows the flow chart of the trial. We will address this question in terms of coprimary outcome measures: antibiotic prescription rate for AECOPD within 30 days post randomisation and the treatment success rate at day 30 post randomisation. Between-group differences in antibiotic prescription rates will be investigated for superiority, while differences in treatment success rates for non-inferiority. The study design follows the Standard Protocol Items: Recommendations for Interventional Trials statement recommendations. ${ }^{18}$ The items from the trial registration data set are recorded in the online supplemental material 1. 


\begin{tabular}{|c|c|}
\hline Inclusion criteria & Exclusion criteria \\
\hline $\begin{array}{l}\text { Hospitalised patients with } \\
\text { AECOPD* }\end{array}$ & $\begin{array}{l}\text { Fever, axillary } \\
\text { temperature } \geq 38^{\circ} \mathrm{C}\end{array}$ \\
\hline$\geq \geq 40$ years of age & $\begin{array}{l}\text { Pneumonia identified by } \\
\text { X-ray or CT of the chest }\end{array}$ \\
\hline $\begin{array}{l}\text { Able to understand and } \\
\text { communicate to ensure } \\
\text { the completion of the trial }\end{array}$ & $\begin{array}{l}\text { Severe respiratory failure } \\
\text { requiring admittance to } \\
\text { ICU } \\
\text { Concurrent infection at } \\
\text { another site (eg, urinary } \\
\text { tract infection) }\end{array}$ \\
\hline \multirow[t]{8}{*}{$\begin{array}{l}\text { Voluntary participation } \\
\text { and provide written } \\
\text { informed consent }\end{array}$} & $\begin{array}{l}\text { Immunosuppression } \\
\text { secondary to } \\
\text { chemotherapy, AIDS or } \\
\text { malignant tumour of blood } \\
\text { system }\end{array}$ \\
\hline & $\begin{array}{l}\text { Comorbidities requiring } \\
\text { corticosteroids (at least } \\
\text { prednisone } 30 \mathrm{mg} / \text { day or } \\
\text { equivalent for more than } \\
30 \text { days) }\end{array}$ \\
\hline & $\begin{array}{l}\text { Invasive mechanical } \\
\text { ventilation }\end{array}$ \\
\hline & $\begin{array}{l}\text { Patients with malignant } \\
\text { tumours receiving } \\
\text { chemotherapy or } \\
\text { radiotherapy }\end{array}$ \\
\hline & - Pregnancy \\
\hline & $\begin{array}{l}\text { Participation in another } \\
\text { clinical trial }\end{array}$ \\
\hline & $\begin{array}{l}\text { Previously enrolment into } \\
\text { the study }\end{array}$ \\
\hline & Refuse to attend \\
\hline
\end{tabular}

*The diagnostic criteria for AECOPD are based on the recommendation in Global Initiative for Chronic Obstructive Pulmonary Disease guideline. ${ }^{4}$

AECOPD, acute exacerbation of chronic obstructive pulmonary disease; AIDS, acquired immunodeficiency syndrome; CT, computer tomography; ICU, intensive care unit.

\section{Population and eligibility criteria}

Recruitment will be conducted at the department of the pulmonary and critical care medicine of the 10 tertiary teaching hospitals in China. The detailed information of these hospitals are shown in online supplemental material 2. The inclusion criteria and exclusion criteria are shown in table 1 . In the context of preventing and controlling COVID-19 pandemic, wearing a mask in whole people in China increase the difficulty of recruitment of eligible patients with AECOPD. According to the current recruitment speed, approximately 30 patients were enrolled into the study every month in 10 centres. We estimate that recruitment duration will last until April 2022.

\section{Informed consent}

Written informed consent (online supplemental material 3) will be required from eligible patients at each participating centres, or their legal representative if they were unable to provide consent.

\section{Randomisation and allocation concealment}

Eligible patients will be randomly assigned (1:1) to either the PCT group or the guideline group within 24 hours after hospitalization. The random sequence was generated by a statistician using SAS software, V.9.4 and stored by a manager from the China-Japan friendship hospital. Once an eligible participant is recruited in each centre, the site investigator will request a random number by telephone from the manager. The investigator receives the group information based on the random number, and then conducted next step according to trial protocol. The participants, healthcare providers and laboratory staff are known to the patient allocation. Outcomes assessors and statisticians will be blinded to the study assignment.

\section{Interventions}

Participants in the PCT group will complete a PCT test within 2 hours after randomisation and the results will be sent back to the clinician by laboratory through the internal network of the hospital. The prescribing clinician will use the results of the PCT to help guide their antibiotic prescription decision. Participants in the guideline group will also need to complete a PCT test within 2 hours after randomisation; however, the laboratory will save the results and do not sent back to the clinician. The detailed antibiotic recommendations of both groups are shown in table 2. Other therapies for AECOPD in both groups will be based on the GOLD

\section{Table 2 Antibiotic prescription strategy in both arms} GOLD guideline-guided antibiotic recommendations

Patients with exacerbations of COPD who have three cardinal symptoms: increase in dyspnoea, sputum volume and sputum purulence; have two of the cardinal symptoms, if increased purulence of sputum is one of the two symptoms; or require mechanical ventilation (invasive or noninvasive).

\section{PCT-guided antibiotic regimen}

\begin{tabular}{ll} 
PCT $<0.1 \mathrm{ng} / \mathrm{mL}$ & $\begin{array}{l}\text { Strongly } \\
\text { discouraged }\end{array}$ \\
$\begin{array}{l}\text { PCT }(0.1-0.25 \mathrm{ng} / \mathrm{mL}) \text { and no sputum } \\
\text { purulence }\end{array}$ & Discouraged \\
$\begin{array}{l}\text { PCT }(0.1-0.25 \mathrm{ng} / \mathrm{mL}) \text { and sputum } \\
\text { purulence }\end{array}$ & Recommended \\
PCT $>0.25 \mathrm{ng} / \mathrm{mL}$ & $\begin{array}{l}\text { Strongly } \\
\text { recommended }\end{array}$ \\
\hline
\end{tabular}

COPD, chronic obstructive pulmonary disease; GOLD, The Global Initiative for Chronic Obstructive Pulmonary Disease; PCT, procalcitonin. 
guideline recommended standard care. All participating centres will be provided the 2020 version of the GOLD guideline.

To ensure the veracity and reliability of results, each centre may adopt any one of the following three validated assays to measure the value of PCT: B.R.A.H.M.S PCT sensitive KRYPTOR assay (Thermo Fisher Scientific, Hennigsdorf, Germany), Roche Elecsys B $\cdot \mathrm{R} \cdot \mathrm{A} \cdot \mathrm{H} \cdot \mathrm{M} \cdot \mathrm{S}$ PCT assay, or the BioMérieux's Vidas B·R·A·H·M.S PCT assay. The direct measuring range of $\mathrm{B} \cdot \mathrm{R} \cdot \mathrm{A} \cdot \mathrm{H} \cdot \mathrm{M} \cdot \mathrm{S}$ PCT sensitive KRYPTOR assay is from 0.02 to $50 \mathrm{ng} / \mathrm{mL}$, and the Functional Assay Sensitivity (FAS) is $0.06 \mathrm{ng} / \mathrm{mL}$, which is 3-fold to 10 -fold above normal mean values. ${ }^{16} 19$ The direct measuring range of Roche Elecsys $\mathrm{B} \cdot \mathrm{R} \cdot \mathrm{A} \cdot \mathrm{H} \cdot \mathrm{M} \cdot \mathrm{S}$ PCT assay is from 0.02 to $100 \mathrm{ng} / \mathrm{mL}$, and the FAS is $0.06 \mathrm{ng}$ / $\mathrm{mL} .{ }^{19}$ The direct measuring range of BioMérieux's Vidas $\mathrm{B} \cdot \mathrm{R} \cdot \mathrm{A} \cdot \mathrm{H} \cdot \mathrm{M} \cdot \mathrm{S}$ PCT assay is $0.05-200 \mathrm{ng} / \mathrm{mL}$, and the FAS is $0.09 \mathrm{ng} / \mathrm{mL}^{19}$ Each centre will perform standard calibration procedures on the instruments and analyse two levels of quality control materials with each sample run. Procedure time for all these assays is less than $30 \mathrm{~min}$. Each participating centre will have access to a KRYPTOR machine, a suitable Vidas or Roche immunoanalyser to expedite the sample analysis and rapidly provide PCT results for guidance in the protocol.

\section{Outcomes}

Primary endpoints

- Antibiotic prescription rate within 30 days post randomisation, expressed as the proportion of patients receiving antibiotics for AECOPD. The objective of antibiotic prescription is to treat AECOPD during hospitalisation or after discharge; antibiotics used for other reasons are excluded, such as new urinary tract infection.

- Treatment success rate at day 30 post randomisation. Treatment success is defined as cure (a complete resolution of signs and symptoms associated with the exacerbation) or improvement (a resolution or reduction of the symptoms and signs associated with the exacerbation, without new symptoms or signs). ${ }^{20}$ Treatment failure is defined as absence of resolution of symptoms and signs; worsening of symptoms and signs; occurrence of new symptoms and signs associated with the primary or with a new infection; or death of any reason after randomisation. ${ }^{20}$ Faceto-face consult will be performed on day 30 post randomisation.

\section{Secondary endpoints}

- Antibiotic prescription rate at day 1 post randomisation.

- Hospital antibiotic exposure, expressed as the number of days of antibiotic consumed for AECOPD and the proportion of patients receiving antibiotic for AECOPD during hospitalisation.

- Length of hospital stay, expressed as the number of days of hospitalisation.
- Rate of subsequent exacerbation, hospital readmission and overall mortality within 30 days post randomisation.

- Change in lung function, COPD assessment test ${ }^{21}$ and modified Medical Research Council dyspnoea scale, ${ }^{22}$ expressed as the difference between the baseline of hospital admission and day 30 post randomisation.

- Intensive care unit admission rate.

\section{Follow-up}

The total follow-up period is 30 days post randomisation. The follow-up items at multiple time points are shown in table 3. Notably, we will contact all the eligible participants and ask them to participate a face-to-face interview at each participating centre on the day 30 post randomisation.If eligible participants can not attend the face-toface interview on day 30, outcome assessors will collect corresponding data via telephone.

\section{Adverse events}

Choice and duration of antibiotics and other pharmacological treatments including bronchodilators and glucocorticoids in both groups are all based on current guideline recommendations. The study intervention will not change the daily clinical treatment therapy, and consequently will not increase the risk of adverse events. Adverse events will be collected and reported as part of routine follow-up. All events fulfilling the definition of a serious adverse event, including death, that occur during the research period will be reported to the research centre expert committee within 24 hours post event occurrence.

\section{Sample size}

This study is designed to have sufficient power to detect a $20 \%$ reduction from an estimated $70 \%$ that consume antibiotics for the AECOPD during the 30 days following randomisation. In the Schuetz's RCT to compare PCT guide antibiotic prescription with guideline therapy, subgroup analysis in patients with AECOPD shown that the 30-day prescription rate of guideline group and PCT group was $69.9 \%$ and $48.7 \%$, respectively. ${ }^{15}$ Detecting a difference in proportions between 0.70 and 0.50 at the $5 \%$ significance level with $90 \%$ power requires a total of 242 participants. Assuming a drop-out rate of $20 \%$, we will need to enrol 302 participants. In addition, we aim to have sufficient power to demonstrate that participants managed with PCT-guided strategy are non-inferior, compared with those managed with guideline recommendations, in terms of treatment success rate at day 30 post randomisation. A limited number of studies have reported the success rate at day 30 after randomisation in patients with AECOPD. ${ }^{1623}$ According to prior trials in terms of hospitalised patients with AECOPD, Stolz et al ${ }^{16}$ reported the short-term treatment success rate as $83.9 \%$ (from 14 to 21 days post randomisation) and Prins $e t a l^{23}$ determined the treatment failure rate at day 30 after randomisation as $20.3 \%$. Based on these data and assuming a treatment success rate of 0.8 at day 30 following randomisation, a 
Table 3 Schedule of assessments and data collection

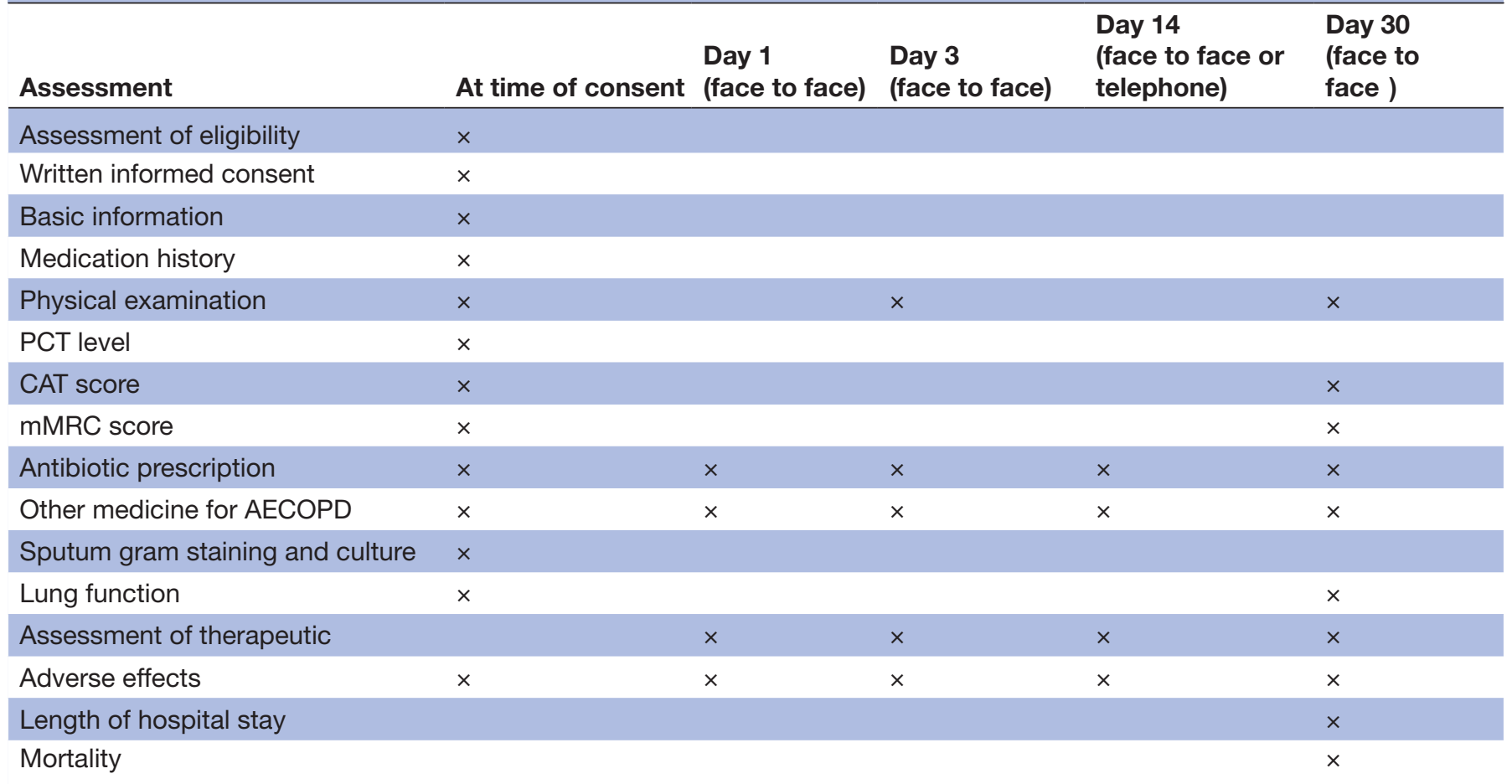

AECOPD, acute exacerbation of chronic obstructive pulmonary disease; CAT, COPD assessment test; ; mMRC, modified Medical Research Council.; PCT, procalcitonin.

non-inferior margin of $0.1,{ }^{24}$ based on a one-sided significance level of $0.05 \%$ and $80 \%$ power, would require 396 participants. Again, with a drop-out rate of $20 \%, 495$ participants will need to be included. Finally, considering these two primary endpoints, we will aim to recruit 500 participants in total to the study.

\section{Data collection and management}

An independent clinician from each centre, unknown to the group information, will collect the data through a prespecified case report form (CRF) at multiple time points. The data filled in the CRF should be accurate, complete, timely and reliable. All centres in our study are qualified by the 'good clinical practice training' of the State Food and Drug Administration for compliance in the training. To increase study awareness and protocol adherence, we will convene principal investigator in each centre and organise a face-to-face meeting to discuss the study protocol before study initiation. Two experienced data managers from the China-Japan hospital biweekly check the CRF of each centre, track clinicians' antibiotic prescription decisions in both groups and assess adherence to the protocol. They will ask investigators to resolve any queries identified, record the reasons for nonadherence and provide regular feedback to every centre. To solve the potential problems and grantee the quality of research, principal investigator at each centre will gather and conduct an online meeting every month. All randomised patients should be followed up until 30 days post randomisation.

\section{Data analysis}

Participants' characteristics and clinical measures will be described by frequencies and percentages, means and SD, or medians and IQRs as appropriate. There will be no planned interim analysis. All analysis will be based on the ITT population, including all randomised participants. Missing data were considered using multiple imputations. All analyses will be completed using SAS, V.9.4 (SAS Institute).

\section{Primary analysis}

- The first primary analysis will aim to compare the rate of antibiotic prescription within 30 days between PCT group and guideline group. A two-sided 95\% CI will be computed for the difference in antibiotic prescription rate for both arms.

- The second primary analysis will compare the difference in proportions of clinical treatment success at day 30 between both groups, relative to a $10 \%$ noninferiority margin, and construct a two-sided $90 \%$ CI for the difference in proportions. Non-inferiority will be declared if the lower limit of the CI exceeds $-10 \%$.

\section{Secondary analysis}

Secondary outcomes will be analysed in a similar manner to the primary outcomes, with linear, logistic and Poisson regression models fitted as appropriate. All tests will be two sided, with $\mathrm{p}$ value less than 0.05 deemed statistically significant. 


\section{Patient and public involvement}

No patients or public involved in the present study. The results of this study will be published by the investigators in relevant scientific peer-reviewed journals, no matter the study findings.

\section{Ethics and dissemination}

This trial has been approved by the Ethics Committee of China-Japan Friendship Hospital (file number: 2020-87 K51). The findings of the study will be published by the investigators in relevant scientific peer-reviewed journals. Once published, the data in our study will be available to other researchers on reasonable request and with the permission of researcher committee.

\section{DISCUSSION}

AECOPD is important event in the management of COPD because it negatively impacts health status, rates of hospitalisation and readmission, and disease progression.

Over $85 \%$ of patients presenting with AECOPD have been prescribed antibiotics in the USA, Europe, and China. ${ }^{5} 67$ However, not all acute exacerbations are driven by bacteria infection, viral infections and environmental factors are currently the main predisposing factors. Current antibiotic prescribing is generally based on the criteria outlined by Anthonisen et al in $1987^{9}$ that include increased dyspnoea, increased sputum volume and increased sputum purulence. The GOLD guideline recommends the use of antibiotics in patients who have all three criteria or, in patients who have two criteria when sputum purulence is one of them, or in patients who require mechanical ventilation. However, whether antibiotic therapy according to Anthonisen criteria will benefit patients is unclear. Some randomised trials have found that only patients with increased sputum purulence benefit from antibiotic therapy with amoxicillin-clavulanate, regardless of the presence or absence of the other two criteria. ${ }^{25}{ }^{26}$ In another randomised trial, doxycycline was not superior to placebo in any Anthonisen criteriadefined subgroup, including among patients with purulent sputum. ${ }^{27}$ Thus, Anthonisen criteria have insufficient diagnostic accuracy to predict which patients can safely be managed without antibiotics.

The dilemma is to identify patients who are most likely to benefit from antibiotics while avoiding unnecessary antibiotic use. PCT, a biomarker of bacterial infection with higher sensitivity and specificity than conventional laboratory tests, ${ }^{28-30}$ would have the potential to distinguish patients in whom antibiotics would be beneficial and guide their duration of use. Due to the small study populations and multiple subgroup's analyses of lower respiratory tract infection in previous studies, ${ }^{13-16}$ it is unclear whether PCT-guided antibiotic therapy is safe and effective for patients with AECOPD. PCT-based protocols may be clinically effective; however, confirmatory trials with rigorous methodology are still required..$^{31}$
In our trial, we aim to evaluate whether PCT-guided antibiotic therapy will reduce the antibiotic prescription rate for patients with AECOPD, in comparison to the GOLD guideline recommendations, without negatively impacting the treatment success rate. If the results of the study are positive (ie, a significant reduction in antibiotic prescribing with no evidence of significant impairment in the treatment success rate), PCT-guided antibiotic therapy is likely to change the guidelines for antibiotic recommendations for patients with AECOPD.

\section{Author affiliations}

${ }^{1}$ Department of Pulmonary and Critical Care Medicine, Capital Medical University, Beijing, China

${ }^{2}$ Department of Pulmonary and Critical Care Medicine,National Center for Respiratory Medicine, Center of Respiratory Medicine, National Clinical Research Center for Respiratory Diseases, China-Japan Friendship Hospital, Beijing, China

${ }^{3}$ Department of Pulmonary and Critical Care Medicine,Beijing Luhe Hospital, Capital Medical University, Beijing, China

${ }^{4}$ Institute of Clinical Medical Sciences, China-Japan Friendship Hospital, Beijing, China

${ }^{5}$ Department of Pulmonary and Critical Care Medicine, Daxing teaching hospital of Capital Medical University, Beijing, China

${ }^{6}$ Institute of Respiratory Medicine, Chinese Academy of Medical Science, Beijing, China

${ }^{7}$ Tsinghua University-Peking University Joint Center for Life Sciences, Beijing, China

Contributors CB, JW and LH conceived and designed the study. YW and WS provided suggestions for the study design. XG performed the sample size calculation. LH and JW drafted and edited the manuscript. All authors have contributed to the revision of the draft and have read and approved the final version.

Funding This study was supported by Chinese Academy of Medical Sciences Innovation Fund for Medical Sciences (CIFMS 2018-12M-1-003 and 2020-I2M-CoV19-005).

\section{Competing interests None declared.}

Patient consent for publication Not required.

Provenance and peer review Not commissioned; externally peer reviewed.

Supplemental material This content has been supplied by the author(s). It has not been vetted by BMJ Publishing Group Limited (BMJ) and may not have been peer-reviewed. Any opinions or recommendations discussed are solely those of the author(s) and are not endorsed by BMJ. BMJ disclaims all liability and responsibility arising from any reliance placed on the content. Where the content includes any translated material, BMJ does not warrant the accuracy and reliability of the translations (including but not limited to local regulations, clinical guidelines, terminology, drug names and drug dosages), and is not responsible for any error and/or omissions arising from translation and adaptation or otherwise.

Open access This is an open access article distributed in accordance with the Creative Commons Attribution Non Commercial (CC BY-NC 4.0) license, which permits others to distribute, remix, adapt, build upon this work non-commercially, and license their derivative works on different terms, provided the original work is properly cited, appropriate credit is given, any changes made indicated, and the use is non-commercial. See: http://creativecommons.org/licenses/by-nc/4.0/.

ORCID iD

Lixue Huang http://orcid.org/0000-0002-9110-9436

\section{REFERENCES}

1 Zhou M, Wang H, Zhu J, et al. Cause-Specific mortality for 240 causes in China during 1990-2013: a systematic subnational analysis for the global burden of disease study 2013. Lancet 2016;387:251-72.

2 Zhong N, Wang C, Yao W, et al. Prevalence of chronic obstructive pulmonary disease in China: a large, population-based survey. $A m \mathrm{~J}$ Respir Crit Care Med 2007;176:753-60. 
3 Wang C, Xu J, Yang L, et al. Prevalence and risk factors of chronic obstructive pulmonary disease in China (the China Pulmonary Health [CPH] study): a national cross-sectional study. Lancet 2018;391:1706-17.

4 Global Initiative for Chronic Obstructive Lung Disease. Global strategy for the diagnosis, management, and prevention of chronic obstructive pulmonary disease. gold, 2020. Available: http:// www. goldcopd.org

5 Lindenauer PK, Pekow P, Gao S, et al. Quality of care for patients hospitalized for acute exacerbations of chronic obstructive pulmonary disease. Ann Intern Med 2006;144:894-903.

6 López-Campos JL, Hartl S, Pozo-Rodriguez F, et al. Antibiotic prescription for COPD exacerbations admitted to hospital: European COPD audit. PLoS One 2015;10:e0124374.

7 Ma Y, Huang K, Liang C, et al. Real-World antibiotic use in treating acute exacerbations of chronic obstructive pulmonary disease (AECOPD) in China: evidence from the ACURE study. Front Pharmacol 2021;12:649884.

8 Butler CC, Gillespie D, White P, et al. C-Reactive protein testing to guide antibiotic prescribing for COPD exacerbations. N Engl J Med 2019;381:111-20.

9 Anthonisen NR, Manfreda J, Warren CP, et al. Antibiotic therapy in exacerbations of chronic obstructive pulmonary disease. Ann Intern Med 1987;106:196-204.

10 Global Initiative for Chronic Obstructive Lung Disease. Global strategy for the diagnosis, management, and prevention of chronic obstructive pulmonary disease. GOLD, 2020.

11 Brusse-Keizer MGJ, Grotenhuis AJ, Kerstjens HAM, et al. Relation of sputum colour to bacterial load in acute exacerbations of COPD. Respir Med 2009;103:601-6.

12 Daniels JMA, de Graaff CS, Vlaspolder F, et al. Sputum colour reported by patients is not a reliable marker of the presence of bacteria in acute exacerbations of chronic obstructive pulmonary disease. Clin Microbiol Infect 2010;16:583-8.

13 Christ-Crain M, Jaccard-Stolz D, Bingisser R, et al. Effect of procalcitonin-guided treatment on antibiotic use and outcome in lower respiratory tract infections: cluster-randomised, single-blinded intervention trial. Lancet 2004;363:600-7.

14 Kristoffersen KB, Søgaard OS, Wejse C, et al. Antibiotic treatment interruption of suspected lower respiratory tract infections based on a single procalcitonin measurement at hospital admission--a randomized trial. Clin Microbiol Infect 2009;15:481-7.

15 Schuetz P, Christ-Crain M, Thomann R, et al. Effect of procalcitoninbased guidelines vs standard guidelines on antibiotic use in lower respiratory tract infections: the ProHOSP randomized controlled trial. JAMA 2009;302:1059-66.

16 Stolz D, Christ-Crain M, Bingisser R, et al. Antibiotic treatment of exacerbations of COPD: a randomized, controlled trial comparing procalcitonin-guidance with standard therapy. Chest 2007;131:9-19.

17 Wang J-X, Zhang S-M, Li X-H, et al. Acute exacerbations of chronic obstructive pulmonary disease with low serum procalcitonin values do not benefit from antibiotic treatment: a prospective randomized controlled trial. Int J Infect Dis 2016;48:40-5.

18 Chan A-W, Tetzlaff JM, Gøtzsche PC, et al. Spirit 2013 explanation and elaboration: guidance for protocols of clinical trials. BMJ 2013;346:e7586.

19 Assink-de Jong E, de Lange DW, van Oers JA, et al. Stop Antibiotics on guidance of Procalcitonin Study (SAPS): a randomised prospective multicenter investigator-initiated trial to analyse whether daily measurements of procalcitonin versus a standard-of-care approach can safely shorten antibiotic duration in intensive care unit patients--calculated sample size: 1816 patients. BMC Infect Dis 2013;13:178.

20 Chow AW, Hall CB, Klein JO, et al. Evaluation of new anti-infective drugs for the treatment of respiratory tract infections. infectious diseases Society of America and the food and drug administration. Clin Infect Dis 1992;15 Suppl 1:S62-88.

21 Jones PW, Harding G, Berry P, et al. Development and first validation of the COPD assessment test. Eur Respir J 2009;34:648-54.

22 Fletcher CM. Standardised questionnaire on respiratory symptoms: a statement prepared and Approved by the MRC Committee on the aetiology of chronic bronchitis (MRC breathlessness score). BMJ 1960;2:1662.

23 Prins HJ, Duijkers R, Lutter R, et al. Blood eosinophilia as a marker of early and late treatment failure in severe acute exacerbations of COPD. Respir Med 2017;131:118-24.

24 Rohde GGU, Koch A, Welte T, et al. Randomized double blind placebo-controlled study to demonstrate that antibiotics are not needed in moderate acute exacerbations of COPD--the ABACOPD study. BMC Pulm Med 2015;15:5.

25 Llor C, Moragas A, Hernández S, et al. Efficacy of antibiotic therapy for acute exacerbations of mild to moderate chronic obstructive pulmonary disease. Am J Respir Crit Care Med 2012;186:716-23.

26 Miravitlles M, Moragas A, Hernández S, et al. Is it possible to identify exacerbations of mild to moderate COPD that do not require antibiotic treatment? Chest 2013;144:1571-7.

27 van Velzen P, Ter Riet G, Bresser P, et al. Doxycycline for outpatienttreated acute exacerbations of COPD: a randomised double-blind placebo-controlled trial. Lancet Respir Med 2017;5:492-9.

28 Assicot M, Gendrel D, Carsin H, et al. High serum procalcitonin concentrations in patients with sepsis and infection. Lancet 1993;341:515-8.

29 Harbarth S, Holeckova K, Froidevaux C, et al. Diagnostic value of procalcitonin, interleukin-6, and interleukin- 8 in critically ill patients admitted with suspected sepsis. Am J Respir Crit Care Med 2001;164:396-402.

30 Simon L, Gauvin F, Amre DK, et al. Serum procalcitonin and Creactive protein levels as markers of bacterial infection: a systematic review and meta-analysis. Clin Infect Dis 2004;39:206-17.

31 Mathioudakis AG, Chatzimavridou-Grigoriadou V, Corlateanu $A$, et al. Procalcitonin to guide antibiotic administration in COPD exacerbations: a meta-analysis. Eur Respir Rev 2017;26. doi:10.1183/16000617.0073-2016. [Epub ahead of print: 31012017 ]. 